\title{
Accuracy of multitarget indirect enzyme-linked immunoassay assay for detection of tuberculosis antibody
}

\author{
Yang Hu ${ }^{1 \#}$, Ming Liu ${ }^{1 \#}$, Haili Hu ${ }^{1 \#}$, Hua Yang ${ }^{1}$, Lianhua Qin ${ }^{1}$, Zhongyi Hu ${ }^{1}$, Changtai Zhu ${ }^{2}$, \\ Zhonghua Liu ${ }^{1}$
}

${ }^{1}$ Shanghai Key Laboratory of Tuberculosis, Clinic and Research Center of Tuberculosis, Shanghai Pulmonary Hospital, Tongji University School of Medicine, Shanghai, China; ${ }^{2}$ Department of Laboratory Medicine, Shanghai Jiao Tong University Affiliated Sixth People's Hospital, Shanghai, China Contributions: (I) Conception and design: Y Hu, M Liu, Z Hu, C Zhu, Z Liu; (II) Administrative support: H Hu, H Yang, L Qin, Zhongyi Hu, Z Liu; (III) Provision of study materials or patients: Y Hu, M Liu, H Hu, H Yang, L Qin, Z Hu, Z Liu; (IV) Collection and assembly of data: Y Hu, M Liu, H Hu, C Zhu, Z Liu; (V) Data analysis and interpretation: Y Hu, M Liu, H Hu, C Zhu, Z Liu; (VI) Manuscript writing: All authors; (VII) Final approval of manuscript: All authors.

\#These authors contributed equally to the work.

Correspondence to: Zhonghua Liu. Shanghai Key Laboratory of Tuberculosis, Clinic and Research Center of Tuberculosis, Shanghai Pulmonary Hospital, Tongji University School of Medicine, Shanghai, China. Email: nllzh@126.com; Changtai Zhu. Department of Laboratory Medicine, Shanghai Jiao Tong University Affiliated Sixth People’s Hospital, No. 600 Yishan Road, Shanghai 200233, China. Email: zct101@163.com.

Background: Diagnosis of tuberculosis (TB) is still difficult. The development of rapid and sensitive laboratory tools for the diagnosis of tuberculosis is a priority. This study aimed to develop an indirect enzyme-linked immunoassay (ELISA) assay for detection of TB antibody and explore its diagnostic value in patients with pulmonary tuberculosis (PTB) via a multi-center clinical evaluation.

Methods: The specific antigen, fusion antigen, and specific antibody peptide were obtained using molecular cloning and phage peptide library screening. An indirect ELISA assay was developed using multiple target materials. Further, the assay was validated in six institutions with clinically confirmed TB patients, non-TB patients with pulmonary disease, and healthy controls as research subjects.

Results: An indirect ELISA assay was developed with $16 \mathrm{kD}$ antigen, 11,488 (CFP10-MPT48-TB8.4) fusion antigen, and TB18 and pl2 as target antigens against TB antibody. The results of this multicenter study showed that the sensitivity, specificity, and the area under the receiver operating characteristic curve (AUC) of the assay were 48.25\% [95\% confidence interval (CI): 45.5-51.1\%], 92.20\% (95\% CI: 90.7-93.5\%) and 0.724 (95\% CI: 0.707-0.741), respectively, and the cut-off value was 0.119 . According to the meta-analysis, the combined ROC was 0.736 (95\% CI: $0.692-0.779), \mathrm{I}^{2}=83.73 \%$. The sensitivity of the sputum-positive PTB group (culture or smear positive) was 58.75\% (95\% CI: 52.96-65.00\%); the sensitivity in sputum-negative group (culture or smear negative) was $37.38 \%$ (95\% CI: $32.71-42.52 \%$ ), respectively; the sensitivity of the sputum-positive group was significantly higher than that of sputum-negative group (OR $=1.57,95 \%$ CI: $1.29-1.92, \mathrm{P}<0.001)$.

Conclusions: Multitarget indirect ELISA assay based on specific-TB antigen, fusion antigen, and antibody peptide is of value for the diagnosis of PTB and can be used as an auxiliary rapid diagnostic tool to improve the sensitivity of sputum-negative TB.

Keywords: Tuberculosis antibody; pulmonary tuberculosis (PTB); indirect enzyme-linked immunoassay (ELISA); diagnostic test; multicenter study

Submitted Oct 08, 2021. Accepted for publication Nov 12, 2021.

doi: $10.21037 / \mathrm{atm}-21-5598$

View this article at: https://dx.doi.org/10.21037/atm-21-5598 


\section{Introduction}

Tuberculosis (TB) is caused by Mycobacterium tuberculosis (MTB). Pulmonary tuberculosis (PTB) is the most common type. Factors such as immune deficiency, drug and alcohol abuse can increase the risk of developing tuberculosis. As an ancient infectious disease, tuberculosis is a serious threat to human health. At present, Asia and Africa have the largest number of TB burden countries in the world. China is one of the high TB burden countries (HBCs), ranking second among 22 HBCs in the world (after India) (1). PTB is the most common type of TB. Difficult diagnosis is one of the most important reasons why TB is hard to control (2-4). At present, bacteriological smear examination and culture are still the main methods in the clinical application of TB etiology examination, but the sensitivity of smear examination is low. Conventional culture takes 4 to 8 weeks, and rapid instrument culture methods (BD BACTEC MGIT960, Becton, Dickinson and Company, Franklin Lakes, NJ, USA; Merier BacT/Alert 3D, Biomerieux, Marcy-l'Étoile, France and other mycobacterium culture detection systems) take 2 to 6 weeks, and such turnaround times do not meet the needs of clinical treatment (5-7). In recent years, some new rapid TB diagnosis and drug resistance detection technologies, such as Xpert MTB/ RIF (Centers for Disease Control and Prevention, Atlanta, GA, USA), linear probe technique $(8,9)$, and interferon$\gamma$-releasing assays (IGRAs) $(10,11)$, have been developed successfully. However, due to their high cost, incomplete drug resistance spectrum detection, or expensive instruments, their clinical application is limited.

The detection of serum antibody in patients with TB is a rapid detection method. In recent years, some antibody assays for the diagnosis of TB based on IgA or/and IgG or/and IgM against immunodominant antigens including MTB Rv2031, the novel polyprotein, genus and speciesspecific antigen shave been used in clinical practice (12-17), but their sensitivity is still not high. The main reason is that the current TB detection assays have singletarget designs. Previous studies have suggested that multiple-antigen approaches may improve detection of $\mathrm{TB}$ $(18,19)$. Therefore, this study intends to use specific antigen protein plus peptide against TB antibody as targets to detect specific TB antibodies in patients' serum to establish a more sensitive method for the diagnosis of TB. We present the following article in accordance with the STARD reporting checklist (available at https://dx.doi.org/10.21037/atm-215598).

\section{Methods}

\section{Ethical statement}

This study was approved by the Ethics Committee of the Shanghai Pulmonary Hospital of the Tongji University School of Medicine (Shanghai, China) (No. K19-060Y). All experiments were performed following the Helsinki Declaration (as revised in 2013). Verbal informed consent was obtained from each participant.

\section{Strains and reagents}

MTB H37Rv standard strain, Escherichia coli db5a, BL21 (DE3), and pet30a vectors were preserved in our laboratory; PMD18-T vector was purchased from Bao Bioengieering Co., Ltd (Dalian, China); instrumental enzymes and reagents: restriction endonuclease, T4 ligase, tap enzyme, isopropyl $\beta$-d-1-thiogalactopyranoside (IPTG), and purification assay were purchased from Takara Bio (Mountain View, CA, USA); HIS-tag purification kit was purchased from Novagen (Madison, WI, USA); horseradish peroxidase (HRP) goat anti-human immunoglobulin $\mathrm{G}(\mathrm{IgG})$ was from Jackson ImmunoResearch (West Grove, PA, USA); and primers were synthesized by a Shanghai bioengineering company and sequenced by a Yingjun bioengineering company.

\section{Target antigen screening}

The process for typing antigen screening was as follows: (I) Cloning of MTB antigen: the website of the National Center for Biotechnology (http://www.ncbi.nlm.nih.gov/) was searched to identify the gene sequence of the H37Rv strain. H37Rv chromosome DNA was used as a template to amplify the corresponding gene fragment. (II) The recombinant protein was collected, purified, and renatured. The recombinant strain was cultured in lysogeny broth (LB) medium to obtain the corresponding antigen protein. The target protein was eluted using urea and imidazole, and then refolded using phosphate buffer saline step by step. (III) Identification of antigenicity of recombinant protein: the immunogenicity of purified protein was verified using SDS-PAGE western blot. For specific methods, refer to our published article $(20,21)$.

\section{Screening of antibody binding peptides}

The purified serum IgG of TB patients was used to screen TB-specific antibody-binding peptide from phage display of 
seven random peptide libraries to obtain antibody-binding peptides of TB18 and Pl2. The main process was as follows: (I) purification of serum IgG from $\mathrm{TB}$ patients and healthy people: the mixed serum samples of $12 \mathrm{~TB}$ patients and 12 healthy people were purified using a protein $\mathrm{A} / \mathrm{g}$ agarose column, and their purity and concentration were assessed using protein electrophoresis and the Bradford method. (II) Screening of phage display peptide library: serum IgG of TB patients was used as a screening target molecule, and $\mathrm{IgG}$ of healthy people was used as an antiscreening target molecule. The random seven peptide library was screened according to the process of adsorption, elution, and amplification. After three rounds of panning, a single phage was selected for amplification, purification, and sequencing. The positive clones were identified using indirect enzymelinked immunoassay (ELISA), and the peptide sequence was obtained. (III) Peptide synthesis and serum samples detection: the serum of TB patients and control serum (non-TB patients and healthy people) were used to evaluate the serological detection value of different peptides to obtain effective antibody-binding peptide and its detection method. For specific methods, refer to our published article $(22,23)$.

\section{Establishment of indirect ELISA assay}

Sixteen KD antigen and fusion antigen 11,488 (CFP10MPT48-TB8.4) were successfully obtained, and antigens were immunogenic. Sixteen KD antigen and fusion antigen 11,488 and high-affinity epitope determinants TB18 and $\mathrm{Pl} 2$ were optimized using an indirect ELISA assay. The indirect ELISA assay was designed according to the principle of the double antigen sandwich method using a multitarget material-coated carrier: the concentration of

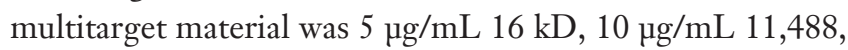
$10 \mu \mathrm{g} / \mathrm{mL}$ TB18, and $10 \mu \mathrm{g} / \mathrm{mL}$ P12; each well was coated with $100 \mu \mathrm{L}$ per plate and left overnight at $4{ }^{\circ} \mathrm{C}$. After washing, it was sealed with blocking liquid containing $5 \%$ skimmed milk powder for $1 \mathrm{~h}$. After washing, $100 \mu \mathrm{L}$ of serum (diluted 5:100 in buffer PBS, ph7.4) were added, and it was incubated at $37^{\circ} \mathrm{C}$ for $1 \mathrm{~h}$. After washing, biotin labeled antibody diluted with 1:10,000 was added, and it was incubated at $37{ }^{\circ} \mathrm{C}$ for $1 \mathrm{~h}$. After washing, $100 \mu \mathrm{L}$ of HRP-labeled avidin were added and it was incubated at $37^{\circ} \mathrm{C}$ for $0.5 \mathrm{~h}$. Tetramethylbenzidine was added for $10 \mathrm{~min}$ at room temperature, and the absorbance value was detected at $450 \mathrm{~nm}$ after termination of the reaction. There were three multiple wells in the experiment, including negative, positive, and blank control.

\section{Source and screening of participants}

The random patients were from six health centers (Center 1: Shanghai Pulmonary Hospital; Center 2: Shanghai Public Health Center; Center 3: Chengdu Tuberculosis Prevention and Control Hospital; Center 4: Hangzhou Red Cross Hospital of the Zhejiang Province; Center 5: Guangxi Longtan Hospital; Center 6: Shanxi Provincial Tuberculosis Prevention and Control Hospital), and the samples were collected from December 2008 to December 2015. The diagnostic criteria of pulmonary TB were: (I) positive smear or culture of TB; or (II) diagnosis of sputumnegative TB with typical pulmonary TB symptoms and chest X-ray manifestations; effective anti-TB treatment; exclusion of other nontuberculous pulmonary diseases after examination; positive antituberculosis antibody in serum; positive polymerase chain reaction (PCR) detection of tuberculous bacteria in sputum; pathological confirmation of TB in lung tissue; and acid-fast mycobacteria were found in the bronchoalveolar lavage fluid. If any of the above three criteria were met, a diagnosis of bacterial-negative TB was made. Exclusion criteria: human immunodeficiency virus (HIV) or immunocompromised patients. The participants in the disease control group were: non-TB patients with various lung diseases, including lung cancer, pneumonia, tracheitis, emphysema, bronchiectasis, etc., all without a TB history. The healthy control group comprised healthy people.

\section{Serological detection of TB antibody}

The serum samples from the six centers were evaluated using an indirect ELISA assay. According to the operating instructions, the laboratory staff of each center conducted independent blind detection and recorded the optical density $(\mathrm{OD})$ values. No clinical information and reference standard results were available to the performers and assessors of the index test.

\section{Statistical analysis}

MedCalc 15.2 was used to calculate the sample size, cutoff value (OD value corresponding to the largest Youden index), sensitivity, specificity, area under receiver operating characteristic curve (AUC), and 95\% confidence interval (CI) of each center and all centers combined. The overall 


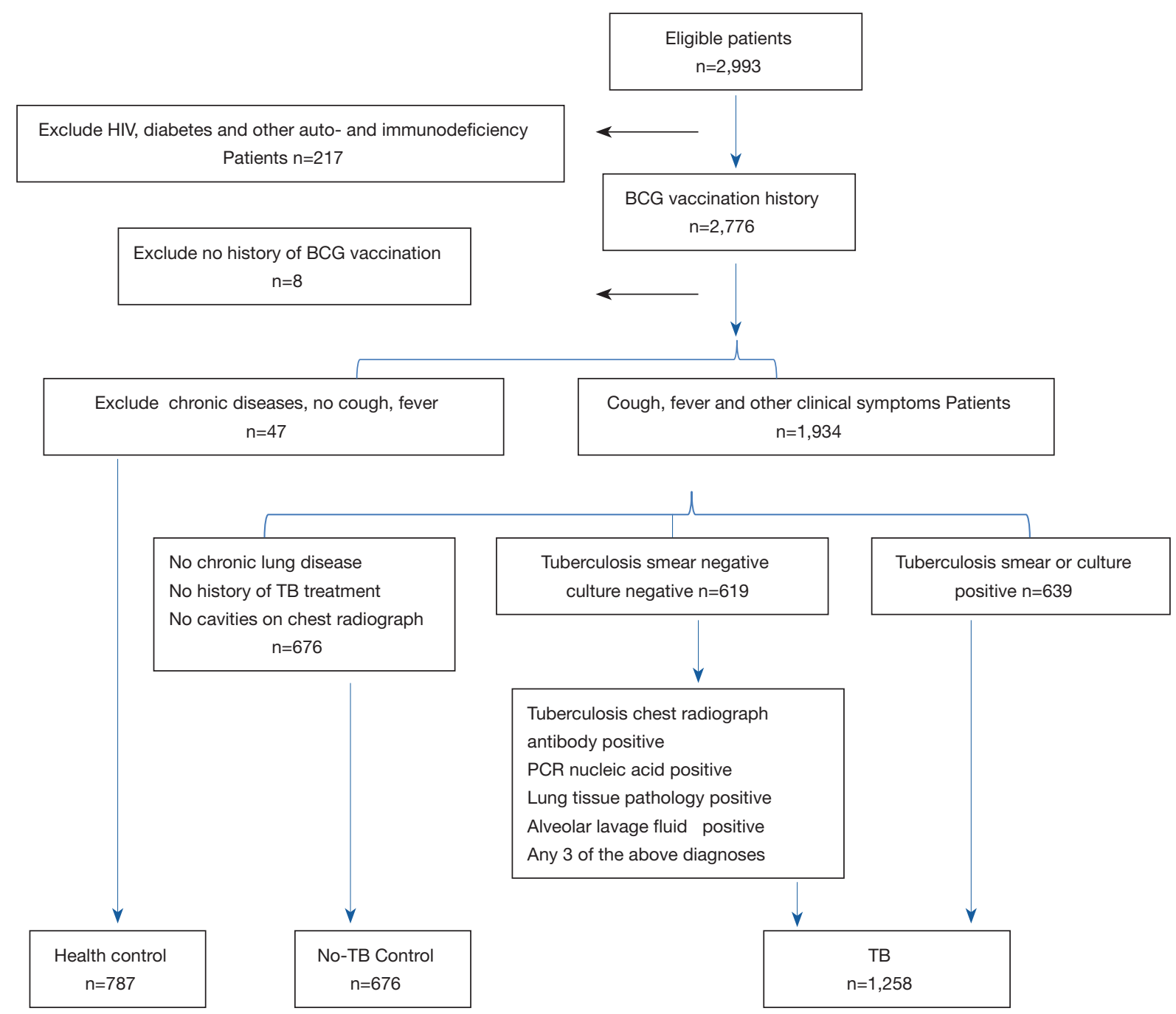

Figure 1 Screening results of included subjects. TB, tuberculosis; No-TB, no tuberculosis.

ROC values were combined using meta-analysis, and the heterogeneity was observed. To observe the threshold effect, the sensitivity and specificity of each center were used for nonparametric correlation analysis. The positive detection rates of the two groups were counted and their values were compared. A $\mathrm{P}<0.05$ was considered as indicating statistical significance.

\section{Results}

\section{Screening process of clinical validation research objects}

The screening process of included studies is shown in Figure 1. A total of 2,721 samples were included in this trial: 1,258 cases in the TB group and 1,463 cases in the control groups (disease control: 676 cases, healthy control group: 787 cases).

\section{Clinical and demographic characteristics of the study population}

The general characteristics of the subjects were as follows: age 43.27-51.5 (interquartile range, IQR), and the proportion of males was $58.57 \%$ (Table 1). The sample size range of the TB group was 112 to 350 , that of the disease control group was 100 to 129 , and that of healthy control group was 90 to 247 (Table 1).

\section{Results of clinical validation}

The validation results of each center showed that the 
Table 1 Clinical and demographic characteristics of the study population

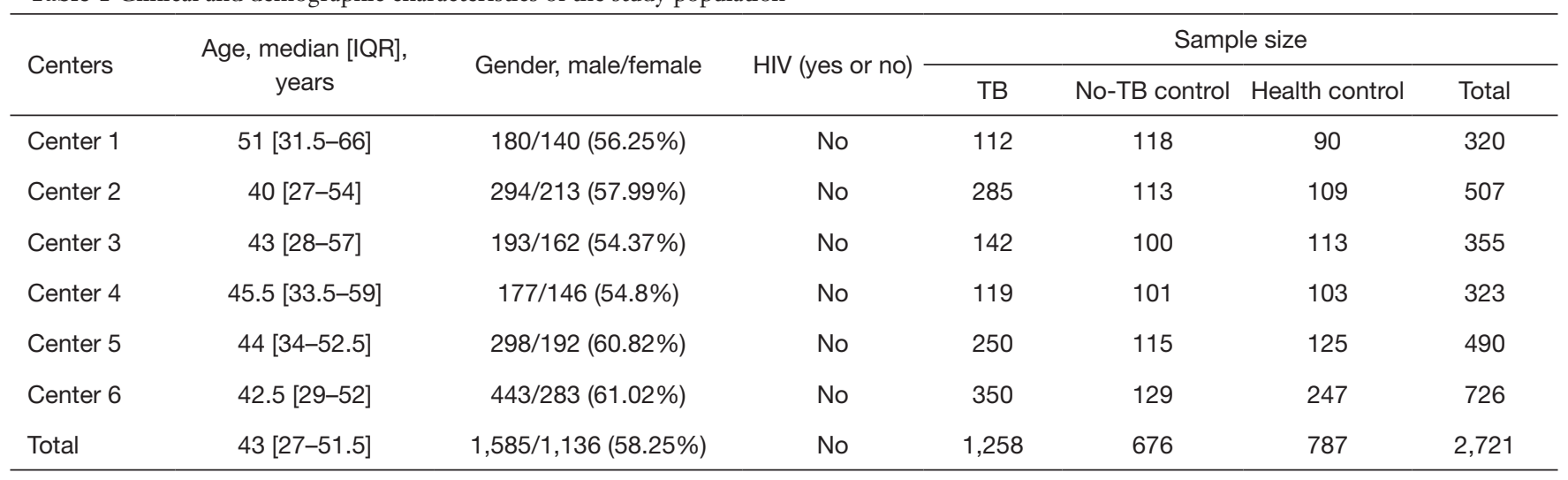

Center 1: Shanghai Pulmonary Hospital; Center 2: Shanghai Public Health Center; Center 3: Chengdu Tuberculosis Prevention and Control Hospital; Center 4: Hangzhou Red Cross Hospital of Zhejiang Province; Center 5: Guangxi Longtan Hospital; Center 6: Shanxi Provincial Tuberculosis Prevention and Control Hospital. HIV, human immunodeficiency virus; TB, tuberculosis.

Table 2 Multicenter clinical validation results of multi-target indirect enzyme-linked immunoassay assay for tuberculosis antibody

\begin{tabular}{lccc}
\hline Centers & Sensitivity $(95 \% \mathrm{Cl})(\%)$ & Specificity $(95 \% \mathrm{Cl})(\%)$ & $\mathrm{AUC}(95 \% \mathrm{Cl})$ \\
\hline Center 1 & $60.71(51.0-69.8)$ & $90.38(85.5-94.0)$ & $0.802(0.754-0.844)$ \\
Center 2 & $43.66(35.4-52.2)$ & $92.96(88.7-96.0)$ & $0.75(0.702-0.794)$ \\
Center 3 & $44.72(38.8-50.7)$ & $96.4(92.4-98.1)$ & $0.658(0.615-0.699)$ \\
Center 4 & $56.00(49.6-62.2)$ & $90.83(86.5-94.2)$ & $0.796(0.757-0.831)$ \\
Center 5 & $52.1(42.8-61.3)$ & $97.06(93.7-98.9)$ & $0.727(0.675-0.775)$ \\
Center 6 & $44.16(38.9-49.5)$ & $90.11(86.6-92.9)$ & $0.686(0.650-0.719)$ \\
Total & $48.25(45.5-51.1)$ & $92.20(90.7-93.5)$ & $0.724(0.707-0.741)$ \\
\hline
\end{tabular}

Center 1: Shanghai Pulmonary Hospital; Center 2: Shanghai Public Health Center; Center 3: Chengdu Tuberculosis Prevention and Control Hospital; Center 4: Hangzhou Red Cross Hospital of Zhejiang Province; Center 5: Guangxi Longtan Hospital; Center 6: Shanxi Provincial Tuberculosis Prevention and Control Hospital. AUC, area under the receiver operating characteristic curve.

sensitivity ranged from $43.66 \%$ to $60.71 \%$, the specificity from $90.11 \%$ to $97.06 \%$, and the AUC from 0.658 to 0.802 (Table 2, Figure 2).

Compared with comprehensive diagnostic criteria, the sensitivity, specificity and AUC of the assay were $48.25 \%$ [95\% confidence interval (CI): 45.5-51.1\%], 92.20\% (95\% CI: 90.7-93.5\%) and 0.724 (95\% CI: 0.707-0.741), respectively, and the cut-off value was 0.119 .

Meta-analysis showed that the AUC of each single center was 0.736 (95\% CI: 0.692-0.779, random effects, $\left.\mathrm{I}^{2}=83.73 \%\right)$, indicating heterogeneity among centers (Figure 3). Spearman's nonparametric correlation analysis showed that the correlation coefficient of the specificity and sensitivity was $-0.0857,95 \% \mathrm{CI}:-0.839$ to $0.780, \mathrm{P}=0.8717$, indicating that there was no threshold effect in this study.

\section{Sensitivity of sputum-positive and sputum-negative groups}

The cut-off value of the assay was 0.118 , and the positive detection rate of the sputum-positive group was $58.75 \%$ (376/640, 95\% CI: 52.96-65.00\%); the sensitivity in the sputum-negative group (culture or smear negative) was $37.38 \%$ (95\% CI: $32.71-42.52 \%$ ), respectively; the sensitivity of the sputum-positive group was significantly higher than that of the sputum-negative group $(\mathrm{OR}=1.57$, 95\% CI: $1.29-1.92, \mathrm{P}<0.001)$.

\section{Adverse events}

There were no adverse events from performing the index test. 

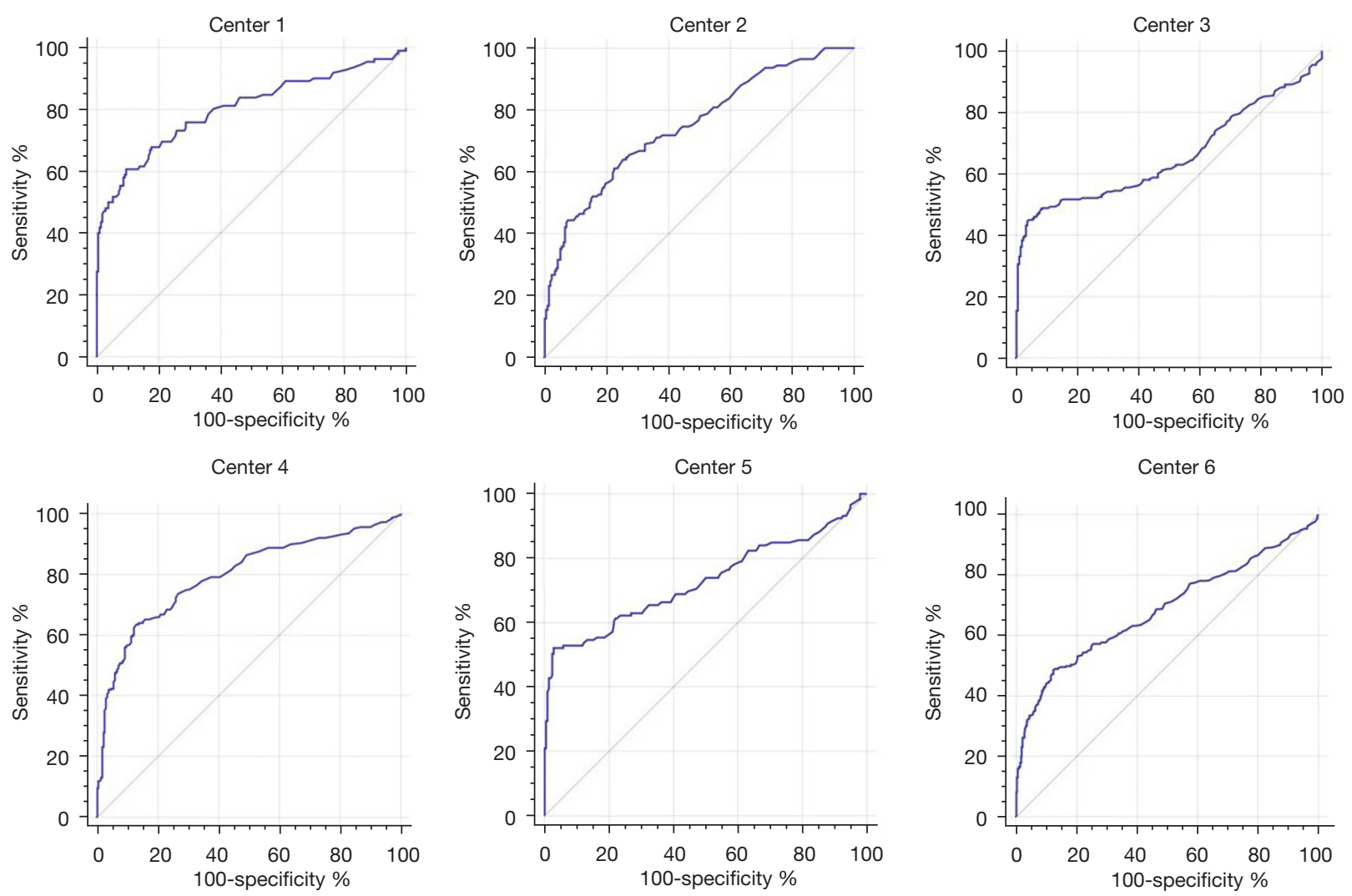

Figure 2 Receiver operating characteristic curves of multicenter clinical validation of multitarget indirect enzyme-linked immunoassay assay for tuberculosis antibody. Center 1: Shanghai Pulmonary Hospital; Center 2: Shanghai Public Health Center; Center 3: Chengdu Tuberculosis Prevention and Control Hospital; Center 4: Hangzhou Red Cross Hospital of the Zhejiang Province; Center 5: Guangxi Longtan Hospital; Center 6: Shanxi Provincial Tuberculosis Prevention and Control Hospital.

\section{Discussion}

Finding a practical and rapid diagnostic tool for TB is a priority. To date, many TB-antibody diagnostic assays have been developed (12-17). However, according to research reports, all assays have been developed based on a single antigen, fusion antigen, epitope, and short peptide determinant. Our previous study showed that there combinant antigen proteins including Rv2031c, Rv0577, and Rv0934 have a certain diagnostic performance, but its value is limited and need be improved (24).

It is clear that there is heterogeneity in TB populations, and some patients are not producing antibodies against certain antigens, which leads to false negative results. To avoid this situation, we selected TB target antigens with high sensitivity to design targets against TB antibodies, and further developed an indirect ELISA method for the serological diagnosis of PTB. The use of a variety of antigens can improve the sensitivity, but each antigen has a determinant epitope that binds to a specific antibody, and each of the epitopes binds to a nonspecific antibody determinant, resulting in a decrease in specificity. To solve this dilemma, we used specific TB antibody-binding peptide to bind with a variety of antigens to block the binding sites of non-specific antigen determinants, so as to improve the specificity.

The validation results of each center show that the sensitivity range of the method is $43.66-60.71 \%$, that of the specificity is $90.11-97.06 \%$, and that of the ROC is $0.658-0.802$. The results of this multicenter validation showed that the sensitivity, specificity, and ROC (95\% CI) of the assay were $48.25 \%$ (95\% CI: $45.5-51.1 \%$ ), $92.20 \%$ (95\% CI: $90.7-93.5 \%)$ and 0.724 (95\% CI: $0.707-0.741 \%)$, respectively, and the cut-off value was 0.119 . These results suggest that the assay is of diagnostic value. 


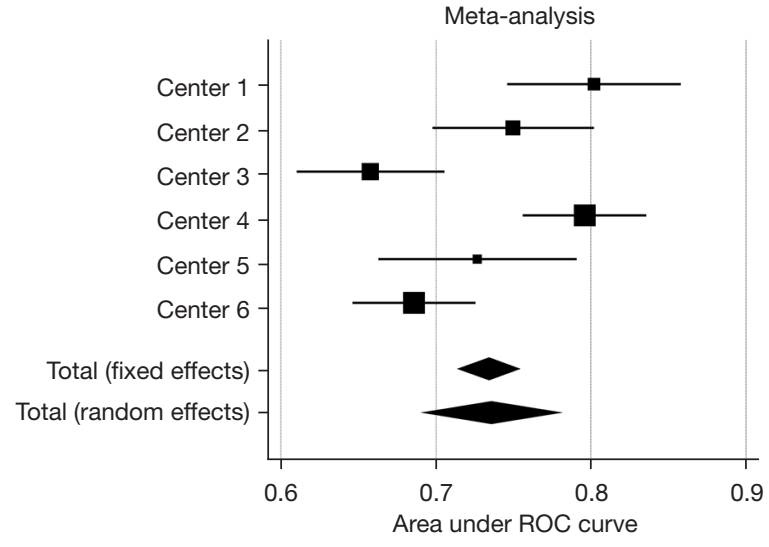

Figure 3 Meta-analysis of area under the receiver operating characteristic curve in multicenter clinical validation of multitarget indirect enzyme-linked immunoassay assay for tuberculosis antibody. Center 1: Shanghai Pulmonary Hospital; Center 2: Shanghai Public Health Center; Center 3: Chengdu Tuberculosis Prevention and Control Hospital; Center 4: Hangzhou Red Cross Hospital of the Zhejiang Province; Center 5: Guangxi Longtan Hospital; Center 6: Shanxi Provincial Tuberculosis Prevention and Control Hospital. AUC, area under the receiver operating characteristic curve; ROC, receiver operating characteristic curve.

According to the meta-analysis, the AUC of each single center was combined. The results showed that the pooled AUC was 0.736 (95\% CI: 0.692-0.779), $\mathrm{I}^{2}=83.73 \%$ (>50\%), indicating that there was significant heterogeneity between the centers. Spearman's correlation analysis showed that there was no threshold effect in this study. The authors believe that different locations and test conditions may jointly affect the results of the evaluation.

The sensitivity in the sputum-negative group (culture or smear negative) was $37.38 \%$ (95\% CI: $32.71-42.52 \%$ ), respectively; the sensitivity of the sputum-positive group was significantly higher than that of the sputum-negative group (OR $=1.57,95 \% \mathrm{CI}: 1.29-1.92)$, suggesting that the production of TB antibodies may be affected by the load of antigen. However, in the sputum-negative group, the assay has also proven valuable in patients with negative TB, which can help to improve the diagnostic rate.

Compared with other methods, indirect ELISA is more rapid and simpler, and it is suitable for most laboratories $(15,24-26)$, and as such it has strong applicability. In this study, although we selected combined multitarget antigens, this approach nonetheless has some limitations (potential bias for selection sample bias and unsatisfactory sensitivity).
At present, tuberculosis complicated with fungal infection is a hot topic. They have similar clinical manifestations (chronic cough, expectoration, intermittent hemoptysis) and similar imaging features. In our study, the controls are mainly pneumonia, bronchitis, emphysema, etc. However, some cases are caused by fungal infection $(27,28)$, we will focus on the differential diagnosis of specific fungal patients in future. Multi-target indirect ELISA is a combination of specific antigen, fusion antigen and antibody binding peptide, and one of its advantages can be applied to detect patients with drug-resistant MTB and with different treatment history. It is clear that antibodies are not affected by these factors. Considering the current approach to the diagnosis of TBs, we recommend this method as an auxiliary diagnostic tool for the diagnosis of pulmonary TB, but the negative results still need to be interpreted carefully.

In summary, multitarget indirect ELISA assay based on specific TB antigen, fusion antigen, and antibody peptide has certain value for the diagnosis of PTB and can be used as an auxiliary rapid diagnostic tool to improve the sensitivity of sputum-negative TB.

\section{Acknowledgments}

We are grateful to Fangyong Yu, Shanghai Lung Hospital; Shuihua Lu, Shanghai Municipal Clinical Center for Public Health; Guangxue Bai, Director of Shanxi Tuberculosis Prevention and Treatment Hospital; Fei Ye, Chengdu Tuberculosis Prevention and Treatment Hospital; Ge He, Hangzhou Red Cross Hospital in Zhejiang Province; and Li Yong, Longtan Hospital in Guangxi for their support with obtaining clinical specimens and related data.

Funding: The work was supported by the Natural Science Foundation of China (81970009).

\section{Footnote}

Reporting Checklist: The authors have completed the STARD reporting checklist. Available at https://dx.doi. org/10.21037/atm-21-5598

Data Sharing Statement: Available at https://dx.doi. org/10.21037/atm-21-5598

Conflicts of Interest: All authors have completed the ICMJE uniform disclosure form (available at https://dx.doi. org/10.21037/atm-21-5598). The authors have no conflicts of interest to declare. 
Ethical Statement: The authors are accountable for all aspects of the work in ensuring that questions related to the accuracy or integrity of any part of the work are appropriately investigated and resolved. This study was approved by the Ethics Committee of Shanghai Pulmonary Hospital of Tongji University School of Medicine (Shanghai, China) (No. K19-060Y). All experiments were performed following the Helsinki Declaration (as revised in 2013). Verbal informed consent was obtained from each participant.

Open Access Statement: This is an Open Access article distributed in accordance with the Creative Commons Attribution-NonCommercial-NoDerivs 4.0 International License (CC BY-NC-ND 4.0), which permits the noncommercial replication and distribution of the article with the strict proviso that no changes or edits are made and the original work is properly cited (including links to both the formal publication through the relevant DOI and the license). See: https://creativecommons.org/licenses/by-nc-nd/4.0/.

\section{References}

1. WHO. Global tuberculosis report 2019. Available online: https://www.who.int/publications/i/item/globaltuberculosis-report-2019

2. Lin M, Liao J, Gong Y, et al. Diabetes and multidrugresistance gene mutation: tuberculosis in Zunyi, Southwest China. Ann Palliat Med 2020;9:3152-61.

3. Tang Y, Shi W, Sun X, et al. Endoscopic ultrasound in diagnosis of esophageal tuberculosis: 10-year experience at a tertiary care center. Dis Esophagus 2017;30:1-6.

4. Raizada N, Sachdeva KS, Nair SA, et al. Enhancing TB case detection: experience in offering upfront Xpert MTB/ RIF testing to pediatric presumptive TB and DR TB cases for early rapid diagnosis of drug sensitive and drug resistant TB. PLoS One 2014;9:e105346.

5. Mekonnen A, Merker M, Collins JM, et al. Molecular epidemiology and drug resistance patterns of Mycobacterium tuberculosis complex isolates from university students and the local community in Eastern Ethiopia. PLoS One 2018;13:e0198054.

6. Duque A, Lin SY, Desmond E, et al. Evaluation of the BD Bactec MGIT 320 system for detection of mycobacteria and drug susceptibility testing of Mycobacterium tuberculosis. J Clin Microbiol 2013;51:3403-5.

7. Zhu C, Cui Z, Zheng R, et al. A multi-center study to evaluate the performance of phage amplified biologically assay for detecting TB in sputum in the pulmonary TB patients. PLoS One 2011;6:e24435.

8. Chen Y, Guo S, Zhao M, et al. Amperometric DNA biosensor for Mycobacterium tuberculosis detection using flower-like carbon nanotubes-polyaniline nanohybrid and enzyme-assisted signal amplification strategy. Biosens Bioelectron 2018;119:215-20.

9. Fang $M, L u$ QR, Hong ZQ. Establishment and application of molecular beacon real-time PCR in the detection of Mycobacteriun tuberculosis. Sichuan Da Xue Xue Bao Yi Xue Ban 2010;41:162-5.

10. Ma Y, Li R, Shen J, et al. Clinical effect of T-SPOT.TB test for the diagnosis of tuberculosis. BMC Infect Dis 2019;19:993.

11. Di L, Li Y. The risk factor of false-negative and falsepositive for T-SPOT.TB in active tuberculosis. J Clin Lab Anal 2018;32:e22273.

12. Abebe F, Belay $M$, Legesse $M$, et al. IgA and IgG against Mycobacterium tuberculosis Rv2031 discriminate between pulmonary tuberculosis patients, Mycobacterium tuberculosis-infected and non-infected individuals. PLoS One 2018;13:e0190989.

13. Broger T, Basu Roy R, Filomena A, et al. Diagnostic Performance of Tuberculosis-Specific IgG Antibody Profiles in Patients with Presumptive Tuberculosis from Two Continents. Clin Infect Dis 2017;64:947-55.

14. Pathakumari B, Prabhavathi M, Anbarasu D, et al. Dynamic IgG antibody response to immunodominant antigens of $M$. tuberculosis for active TB diagnosis in high endemic settings. Clin Chim Acta 2016;461:25-33.

15. Feng X, Yang X, Xiu B, et al. IgG, IgM and IgA antibodies against the novel polyprotein in active tuberculosis. BMC Infect Dis 2014;14:336.

16. Omrani M, Ansari MH, Agaverdizadae D. PCR and Elisa methods (IgG and $\operatorname{IgM}$ ): their comparison with conventional techniques for diagnosis of Mycobacterium tuberculosis. Pak J Biol Sci 2009;12:373-7.

17. Butt T, Malik HS, Abbassi SA, et al. Genus and speciesspecific IgG and IgM antibodies for pulmonary tuberculosis. J Coll Physicians Surg Pak 2004;14:105-7.

18. Dai Z, Liu Z, Xiu B, et al.A multiple-antigen detection assay for tuberculosis diagnosis based on broadly reactive polyclonal antibodies. Iran J Basic Med Sci 2017;20:360-7.

19. Zhu C, Liu J, Ling Y, et al. Evaluation of the clinical value of ELISA based on MPT64 antibody aptamer for serological diagnosis of pulmonary tuberculosis. BMC Infect Dis 2012;12:96. 
20. Wang Q, Yang H, Jin RL, et al. Expression and serodiagnosis of recombinant protein Rv0222 from Mycobacterium tuberculosis. Chinese Journal of Antituberculosis 2012;34:36-9.

21. Yang H, Zhang LT, Li LQ, et al. Screening of the binding peptides of Mycobacterium tuberculosis H37 Rv. Chinese Journal of Tuberculosis And Respiratory Diseases 2011;34:192-6.

22. Yang HS, Hu ZY, Liu ZH, et al. Screening of tuberculosis specific antibody binding peptides. Zhonghua Yu Fang Yi Xue Za Zhi 2011;45:12-6.

23. Tan Y, Dong G, Niu J, et al. Development of an indirect ELISA based on glycoprotein B gene for detecting of Feline herpesvirus type 1. Pol J Vet Sci 2019;22:631-3.

24. Ma G, Pei W, Yang $Y$, et al. emPAI-assisted strategy enhances screening and assessment of Mycobacterium tuberculosis infection serological markers.Microb Biotechnol2021;14:1827-1838.

Cite this article as: $\mathrm{Hu} \mathrm{Y}$, Liu M, Hu H, Yang H, Qin L, Hu Z, Zhu C, Liu Z. Accuracy of multitarget indirect enzyme-linked immunoassay assay for detection of tuberculosis antibody. Ann Transl Med 2021;9(23):1731. doi: 10.21037/atm-21-5598
25. Bonfini B, Tittarelli M, Luciani M, et al. Development of an indirect ELISA for the serological diagnosis of dourine. Vet Parasitol 2018;261:86-90.

26. Laborde JM, Sguazza GH, Fuentealba NA, et al. Indirect ELISA (iELISA) for routine detection of antibodies against Minute Virus of Mice (MVM) in mice colonies. Rev Argent Microbiol 2017;49:210-5.

27. Amiri MRJ, Siami R, Khaledi A, et al. Tuberculosis Status and Coinfection of Pulmonary Fungal Infections in Patients Referred to Reference Laboratory of Health Centers Ghaemshahr City during 2007-2017. Ethiop J Health Sci 2018;28:683-90.

28. Kamwiziku GK, Makangara JC, Orefuwa E, et al. Current burden of serious fungal infections in Republic of Congo. Mycoses 2020;63:543-52.

(English Language Editor: B. Meiser) 